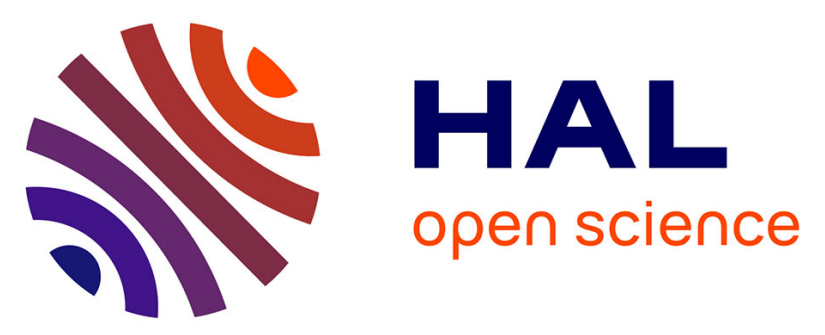

\title{
Modelling and optimization of a floating triangular platform used for nano and microforces sensing.
}

Ali Cherry, Joël Abadie, Emmanuel Piat

\section{To cite this version:}

Ali Cherry, Joël Abadie, Emmanuel Piat. Modelling and optimization of a floating triangular platform used for nano and microforces sensing.. IEEE/RSJ International Conference on Intelligent Robots and Systems, IROS'07., Oct 2007, San Diego, CA., United States. pp.1118-1123. hal-00186818

\section{HAL Id: hal-00186818 https://hal.science/hal-00186818}

Submitted on 12 Nov 2007

HAL is a multi-disciplinary open access archive for the deposit and dissemination of scientific research documents, whether they are published or not. The documents may come from teaching and research institutions in France or abroad, or from public or private research centers.
L'archive ouverte pluridisciplinaire HAL, est destinée au dépôt et à la diffusion de documents scientifiques de niveau recherche, publiés ou non, émanant des établissements d'enseignement et de recherche français ou étrangers, des laboratoires publics ou privés. 


\title{
Modelling and optimization of a floating triangular platform used for nano and microforces sensing
}

\author{
A. Cherry, J. Abadie and E. Piat
}

\begin{abstract}
This paper presents the dynamic behaviour modelling of a horizontal triangular platform, used as the sensing part of a microforce sensor. This sensor is based on a magnetic and a buoyancy principle. A particular configuration used to obtain a linear model is presented. This linear model will be essential for the futur control of the system, in order to achieve force measurement without displacement of the platform. The determination of the platform position and orientation in the horizontal plane is done thanks to three laser range sensors. The sensors configuration provide a linear transformation between the three measured ranges and the position and orientation of the platform. Finally, an open loop result comparison is done between the linear state model and a more complex 3D non linear model.
\end{abstract}

\section{INTRODUCTION}

Manipulation with force control is an emerging area that appears certain to become an important component in microsystems technology. Pure position control is sometimes not suitable to ensure successful operation and prevent damage to the manipulated micro objects. Force control is often needed in order to achieve better manipulation results [6]. Moreover, in some specific applications like biological cells characterization, obtaining force information is a main objective [7] [8].

This paper presents the 2D modelling of a floating triangular platform which is the sensing part of a new magnetic nano and microforce sensor for a large field of aplications (force identification, stiffness characterization, micro-assembly with force control,...). The platform on which the object to be handled or characterized is locked, presents a naturally stable six degrees of freedom equilibrium position using the combination of upthrust buoyancy and magnetic forces. The magnetic stiffness is typically between $10 \mathrm{nN} / \mu \mathrm{m}$ and 50 $n N / \mu m$.

This paper presents the differential equations which govern the platform. In order to simplify these equations and to obtain a 2D linear model, a particular configuration of the magnetic and sensing parts have been studied. The $2 \mathrm{D}$ model is essential for the control of the system in order to achieve forces measurement without displacement of the platform. In this paper a way to calculate the platform position using the combination of three laser range sensors is also presented.

\section{FORCE MEASUREMENT PRINCIPLE}

Forces sensing is strongly related to the measurement of a rigid microstructure displacement or the deformation

This work was not supported by any organization

A. Cherry, J. Abadie and E. Piat are with Laboratoire d'automatique de Besançon, UFC, ENSMM, CNRS ; 24, rue Alain Savary, 25000 Besançon, France. Emmanuel.piateens 2 m. fr of an elastic structure [1]. The applied force is directly calculated using the structure stiffness which is established after calibration. Generally, calibration is a problem for micro and nano force sensors because of the lack of standard at this scale. Thus, calibration must be performed using indirect approaches.

The dry friction is the major problem for this measurement approach. Generally, micro and nano force sensors use elastic micro structures like micro beam to avoid dry friction [6]. Another way to avoid friction problem, is the use of levitation methods. In this case the absence of friction associated with a low stiffness makes the sensor highly sensitive. The only problem is that passive levitation is unstable. This result was shown theoretically for electrostatic case by Earnshaw in 1841 [5]. Attractive forces like magnetic forces coupled with repulsive forces like diamagnetic forces are needed to achieve passive levitation. A passive micro and nano force sensor using magnetic and diamagnetic forces was presented in [3].

In the new sensor presented here a special attention has also been devoted to the magnetic levitation approach. We decided also to use a floating principle rather than diamagnetic forces in order to stabilize the passive magnetic levitation. This principle allows to suppress the weight limitation of the levitating part (see figure 1).
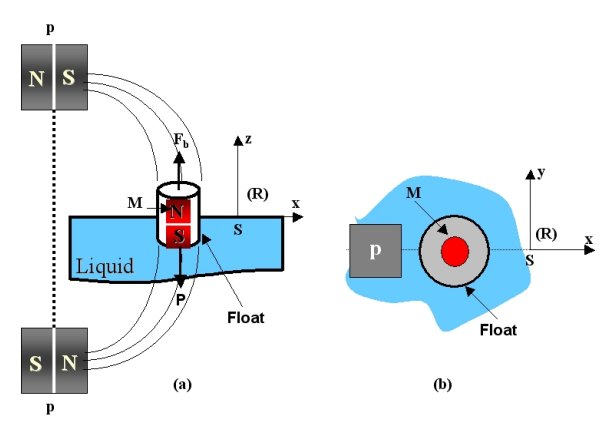

Fig. 1. (a) Floating-magnetic principle (b) Top view

With such a configuration, magnetic forces of the external magnets $p$ provide stability in the plane $(x O y)$ of the floating part inside which a small magnet $M$ is fixed. The upthrust buoyancy acting against the gravity provides stability along $\vec{z}$ axis. In the plane $(x O y)$ the upthrust buoyancy has no action. Thus, the external forces in the plane $(x O y)$ applied to the floating part can be deduced according to the floating part magnetic stiffness and the displacement measured by a 
laser beam.

This principle of force measurement related to the sensing part displacement of the microforce sensor can generate drawbacks in the case of high precision tasks such as biological cells micromanipulation or micro-assembling, where the manipulated object should not move. Thus, the key idea here is to developp a device wich allows force measurements without any (or only very small) displacements of the sensitive part, thanks to an active control. The design used to make possible the feedback control loop is composed of two coils placed on both sides, above and below the floating magnet (see figure 2).

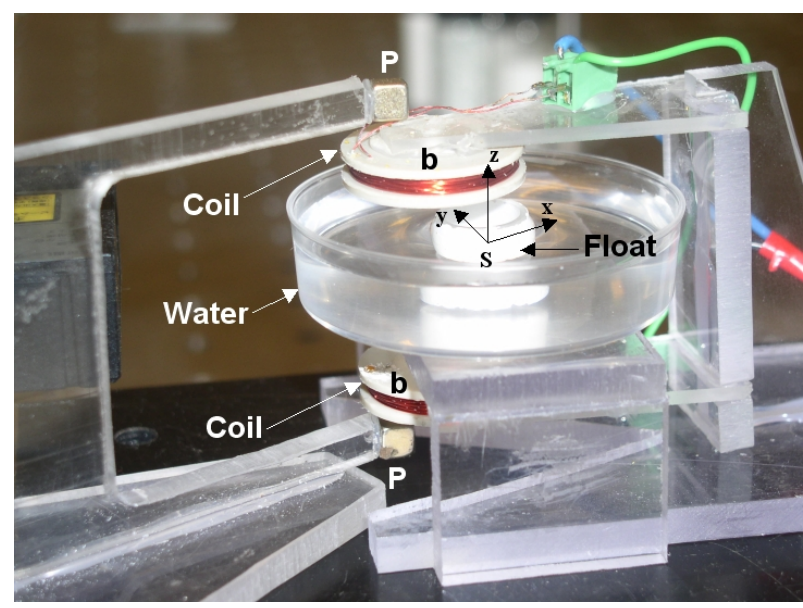

Fig. 2. Floating mechanism equipped with two coils

In case of an external force applied to the sensing element which tends to move it, the activation of coils will produce an opposed electromagnetic force $\overrightarrow{F e l e c}$ that will maintain the sensing element on its initial position. In this case, the current $I$ in the coils is the new physical value related to the external force. The complete study of this force measurement principle and experimental validation are presented in [4].

\section{Stability In The Plane $(x O y)$}

In this section, the study will be focused on the stability in the plane $(x O y)$. The configuration considered is described in figure 2. We are not going to demonstrate mathematically this stability, but we will illustrate it with a simulation. The figure 3 shows the force $\vec{F}$ vectors applied on the float, which is the sum of the magnetic force $F^{\overrightarrow{m a g}}$ generated by the two fixed cubic magnets $p$ (represented by a square) and the electromagnetic force $F \overrightarrow{e l e c}$ generated by the two coils $b$ (represented by a dotted circle). We can see clearly, when no current is applied in the coils $(i=0)$, the stable equilibrium position $S$ of the moving cylindrical magnet $M$ at $x=0.0115, y=0$ and $z=0$. At this point, the magnetic force generated by $p$ is null. A current $i$ different from zero doesn't disturb the stability in the plane but changes the position of $S$ (see figure 3 ). $S_{i}$ is the new stable equilibrium position for a non null current $i$.
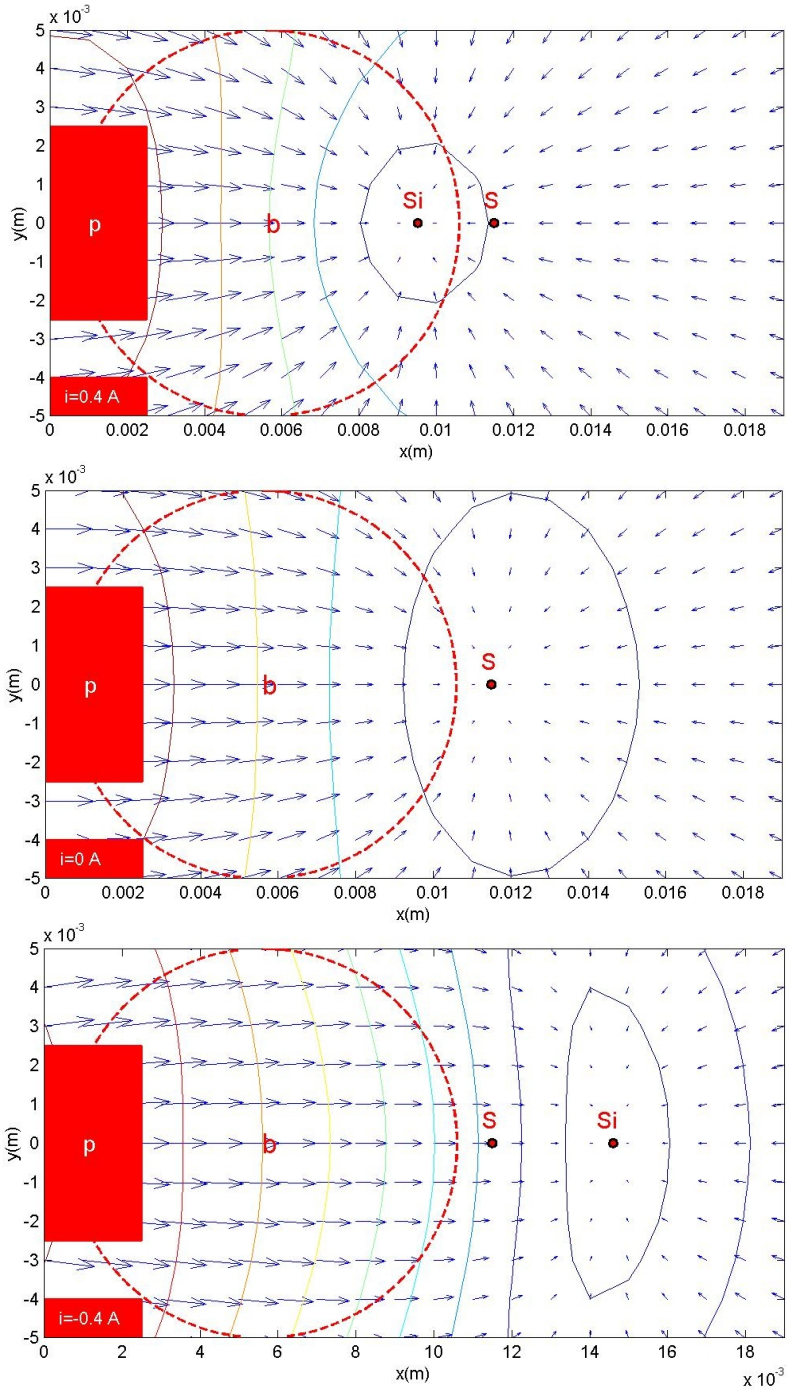

Fig. 3. Force $\vec{F}$ vectors in the plane $(x O y)$ for $i=0.4 A, i=0 A$ and $i=-0.4 A$

It remains to note that in case of small displacements around the point of equilibrium $S$, the magnetic force $\vec{F} \overrightarrow{m a g}$ is quasi linear [2] and we can write :

$$
F_{/ R}^{\overrightarrow{m a g}}=K_{m a g} \cdot \vec{M}_{/ R}=\left[\begin{array}{cc}
K_{m}^{x} & 0 \\
0 & K_{m}^{y}
\end{array}\right] \cdot \vec{M}_{/ R}
$$

where $R$ is the reference frame of $(S, \vec{x}, \vec{y})$ shown in figure 1 and $M^{T}=\left[x_{M}, y_{M}\right]$ the position of the moving cylindrical magnet $M$ in the plane (xOy).

Around $S$, the electromagnetic force is proportionnal to the current $I$ in the coils [4] and we can write :

$$
F_{/ R}^{\overrightarrow{e l e c}}=K_{\text {elec }} \cdot i=\left[\begin{array}{c}
K_{e} \\
0
\end{array}\right] \cdot i
$$

\section{2D PLATFORM}

In the following, the floating-magnetic principle described in section (II) will be used in order to construct a force 
measurement device which will have, as a sensing element, a floating platform on which the object to be manipulated is locked (see figure 4). The platform mass is suspended against gravity by the combined upthrust buoyancy of three floats placed at the corners of the platform. Thus, the platform weight is not an issue for this sensor. Each degree of freedom in the plane $(x O y)$ can constitute a direction of force or couple measurement.

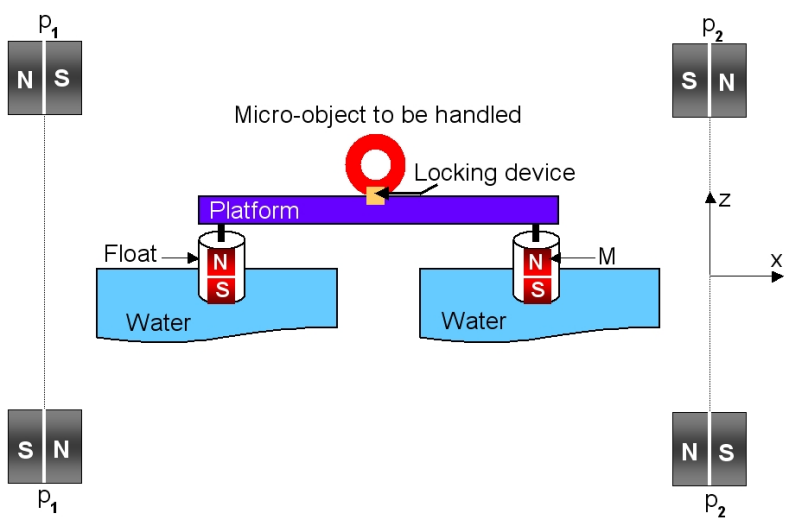

Fig. 4. Platform

\section{A. Analytical expression of $\vec{F}$}

The figure 5 represents the elementar configuration of the platform, including only one couple of magnet $p$ and coils $b$ oriented with an angle $\alpha$ and generating a force $\vec{F}$ on the cylindrical magnet $M$ placed at the corner of the moving platform. $R_{1}$ is the reference frame of $\left(S_{1}, \overrightarrow{x_{1}}, \overrightarrow{y_{1}}\right)$, with $S$ the stable equilibrium position of $M$. The platform is moving freely according to a fixed reference frame $R_{0}\left(O, \overrightarrow{x_{0}}, \overrightarrow{y_{0}}\right)$. We also define a reference frame $R_{p}\left(G, \overrightarrow{x_{p}}, \overrightarrow{y_{p}}\right)$ related to the platform with :

$$
G=\left[\begin{array}{l}
X \\
Y
\end{array}\right]_{/ R_{0}} \text { et }\left(\overrightarrow{x_{0}}, \overrightarrow{x_{p}}\right)=\psi
$$

$X$ and $Y$ are the coordinates of $G$ in the reference frame $R_{0}$.

When the platform is on its initial position, both reference

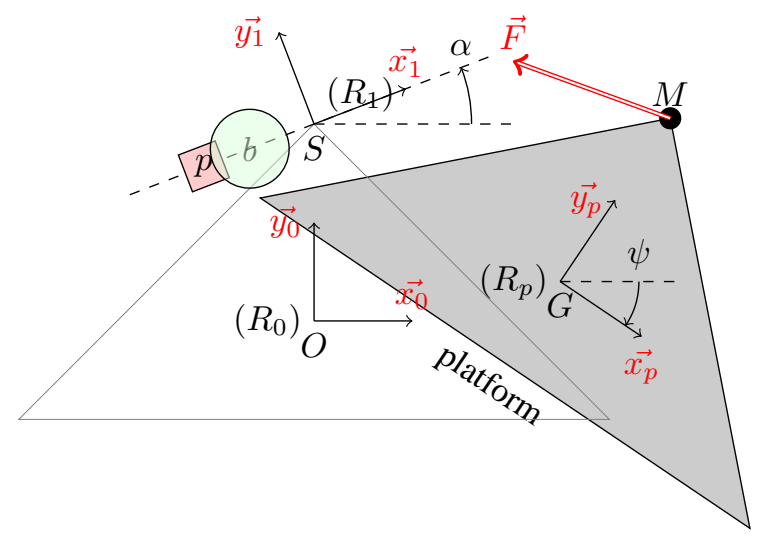

Fig. 5. Platform simple configuration (top view) frames $R_{0}$ and $R_{p}$ are superimposed. $M$ and $S$ are also superimposed.

\section{B. Change of reference frame}

The reference frame change for a point $M$ belonging to the platform, in the general case, is done by the following transformation :

$$
M_{/ R_{1}}=P_{10}\left[\left(P_{0 p} \cdot M_{/ R_{p}}\right)+R_{p}-R_{1}\right]
$$

$P_{01}$ et $P_{0 p}$ are the crossing matrices from $R_{1}$ to $R_{0}$ and from $R_{p}$ to $R_{0}$ and they have the following expressions:

$$
P_{10}=\left[P_{01}\right]^{-1}=\left[\begin{array}{cc}
\cos \alpha & \sin \alpha \\
-\sin \alpha & \cos \alpha
\end{array}\right], P_{0 p}=\left[\begin{array}{cc}
\cos \psi & \sin \psi \\
-\sin \psi & \cos \psi
\end{array}\right]
$$

in which $\alpha$ is the angle formed by the two reference axes $\overrightarrow{x_{1}}$ and $\overrightarrow{x_{0}}$ and $\psi$ is the angle formed by the two reference axes $\overrightarrow{x_{p}}$ and $\overrightarrow{x_{0}}$.

The reference frame change for a force vector $\vec{F}$, under the same conditions, is done by the following transformation:

$$
\overrightarrow{F_{/ R_{0}}}=P_{01} \cdot \overrightarrow{F_{/ R_{1}}}
$$

\section{Force $\vec{F}$ applied on $M$}

In this section, we give the expression of the force $\vec{F}$ applied on $M$ in $R_{0}$. This force is the sum of the magnetic force $F^{\overrightarrow{m a g}}$ due to $p$ and the electromagnetic force $F \overrightarrow{e l e c}$ due to the coil $b$ applied to the moving cylindrical magnet $M$ placed at the corner of the platform. For small displacements around $S_{1}$, we have:

1) Magnetic force: According to equations (1), (3) and (4):

$$
F_{/ R_{0}}^{\overrightarrow{m a g}}=P_{01} \cdot K_{m a g} \cdot P_{10}\left[\left(P_{0 p} \cdot M_{/ R_{p}}\right)+R_{p}-R_{1}\right]
$$

thus:

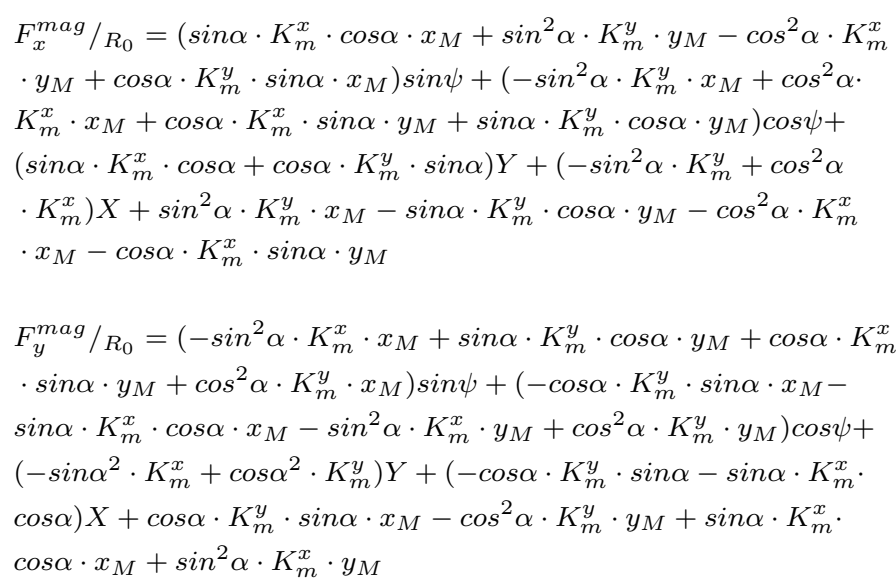

with:

$$
S_{/ R_{0}}=M_{/ R_{p}}=\left[\begin{array}{l}
x_{M} \\
y_{M}
\end{array}\right]
$$




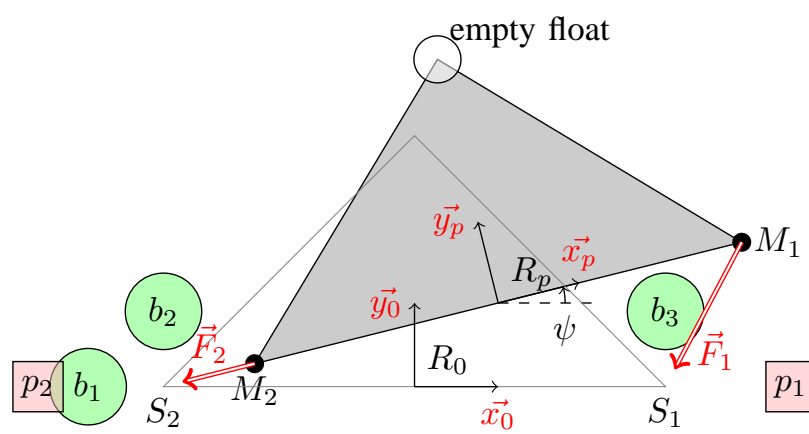

Fig. 6. Platform particular configuration

2) Electromagnetic force: According to equations (2), (3) and (4):

$$
F_{/ R_{0}}^{\overrightarrow{e l e c}}=P_{01} \cdot K_{\text {elec }} \cdot i_{1}
$$

We can see easily that the expression of $\vec{F}$ is not linear. So the idea was to use a particular configuration of the platform in order to simplify the differential equations which govern its movement.

\section{Platform particular configuration}

The figure 6 represents the selected configuration for the platform with 3 pairs of coils $b_{i}, 2$ pairs of fixed cubic magnets $p_{i}(5 \times 5 \mathrm{~mm})$ and 2 cylindrical moving magnets $M_{1}$ and $M_{2}$ placed at the corners of the triangular platform, inside a float. An empty float is placed at the third corner in order to ensure the stability of the triangular platform along $\vec{z}$ axis.

This configuration was retained in order to simplify to the maximum the differential equations which govern the movement of the platform and to limitate the coupling between the various directions of measurement (see section V). To each coil $b_{i}$ a reference frame $R_{b_{i}}$ is associated. To each pair of fixed cubic magnet a reference frame $R_{p_{i}}$ is associated. The stable equilibrium position $S_{1}$ of $p_{1}$ is the origin of the reference frame $R_{p_{1}}$ and the stable equilibrium position $S_{2}$ of $p_{2}$ is the origin of the reference frame $R_{p_{2}}$, with:

$$
S_{1 / R_{0}}=M_{1 / R_{p}}=\left[\begin{array}{l}
\delta \\
0
\end{array}\right] \text { and } S_{2 / R_{0}}=M_{2 / R_{p}}=\left[\begin{array}{c}
-\delta \\
0
\end{array}\right]
$$

\section{Platform POSITION}

The determination of the external force $F^{e x t}$ in the plane $(x O y)$ is conditioned by the determination of the magnetic forces $F_{p_{1}}^{\overrightarrow{m a g}}$ and $F_{p_{2}}^{\overrightarrow{m a g}}$ applied on $M_{1}$ and $M_{2}\left(F_{b_{1}}^{\overrightarrow{\text { elec }}}, F_{b_{2}}^{\overrightarrow{\text { elec }}}\right.$ and $F_{b_{3}}^{\overrightarrow{e l e c}}$ are known thanks to the currents $i_{1}, i_{2}$ and $i_{3}$ in the coils $b_{1}, b_{2}$ and $b_{3}$ ). The determination of these magnetic forces is possible knowing the position of the platform. In this section we present the way to calculate the platform position $(X, Y, \psi)$ using three laser range sensors. An elementary configuration with only one laser sensor, used to measure the distance of a deflector fixed on the platform (on

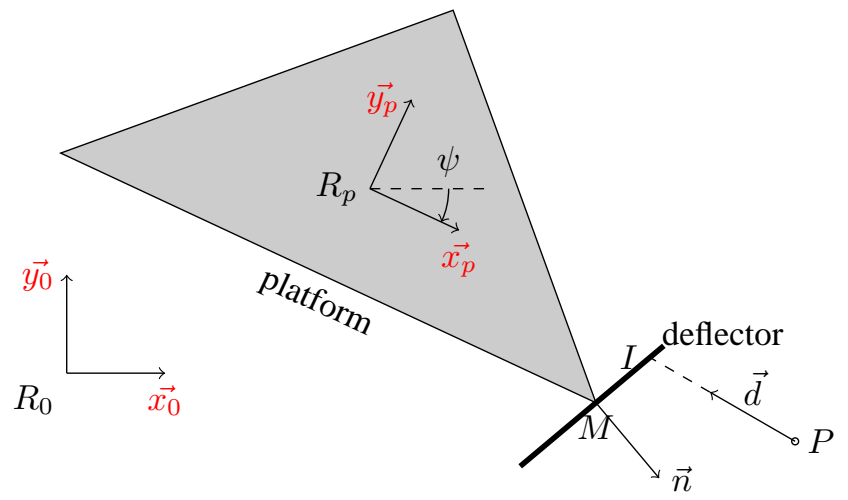

Fig. 7. Elementary configuration of laser sensor (top view)

$M$ ), is first presented (see figure 7). The distance measured by the laser is given by the following expression:

$$
l=\frac{\left.\vec{n}_{/ R_{0}} \cdot P \vec{M}\right|_{/ R_{0}}}{\vec{n}_{/ R_{0}} \cdot \vec{d}_{/ R_{0}}} \quad \text { with }: \overrightarrow{P I}=l \cdot \vec{d}
$$

with:

$$
\left\{\begin{array}{l}
P: \text { origin of measurement } \\
\vec{d}: \text { laser direction } \\
I: \text { laser beam target on the deflector } \\
\vec{n}: \text { normal to the deflector surface } \\
l: \text { distance between } P \text { and } I
\end{array}\right.
$$

thus:

$$
l=\frac{\left(P_{01} \cdot \vec{n}\right) \cdot\left(P_{01} \cdot M+R_{p}-P\right)}{\left(P_{01} \cdot \vec{n}\right) \cdot \vec{d}}
$$

where:

$$
\begin{gathered}
P_{01}=\left(\begin{array}{cc}
\cos \psi & -\sin \psi \\
\sin \psi & \cos \psi
\end{array}\right), R_{p}=\left[\begin{array}{l}
X \\
Y
\end{array}\right]_{/ R_{0}}, \vec{d}=\left[\begin{array}{l}
x_{d} \\
y_{d}
\end{array}\right]_{/ R_{0}} \\
P=\left[\begin{array}{l}
x_{P} \\
y_{P}
\end{array}\right]_{/ R_{0}}, \vec{n}=\left[\begin{array}{l}
x_{n} \\
y_{n}
\end{array}\right]_{/ R_{p}}, A=\left[\begin{array}{l}
x_{A} \\
y_{A}
\end{array}\right]_{/ R_{p}}
\end{gathered}
$$

The developement of the equation (7) allows us to write:

$$
\begin{aligned}
l & =\frac{\left(x_{n} \cos \psi-y_{n} \sin \psi\right) X+\left(x_{n} \sin \psi+y_{n} \cos \psi\right) Y}{\left(y_{d} x_{n}-x_{d} y_{n}\right) \sin \psi+\left(x_{d} x_{n}+y_{d} y_{n}\right) \cos \psi} \\
& +\frac{\operatorname{det}[\vec{P}, \vec{n}] \sin \psi-(\vec{n} \cdot \vec{P}) \cos \psi+\vec{n} \cdot \vec{A}}{\left(y_{d} x_{n}-x_{d} y_{n}\right) \sin \psi+\left(x_{d} x_{n}+y_{d} y_{n}\right) \cos \psi}
\end{aligned}
$$

with $\operatorname{det}[\vec{P}, \vec{n}]=\left(y_{n} x_{P}-x_{n} y_{P}\right)$.

With 3 laser sensors, the determination of the platform position $(X, Y, \psi)$ is possible only if the 3 corresponding equations (8) are inversible.

Because (8) is not linear, an idea to determine $(X, Y, \psi)$ is to use a particular configuration which makes (8) linear.

\section{A. Particular configuration}

The selected configuration is as follows (see figure 8): $\vec{d}$ and $\vec{n}$ are colinear with a norm equal to one and $\vec{n}$ is perpendicular to $\vec{P}$ and $\vec{A}$, Thus: 


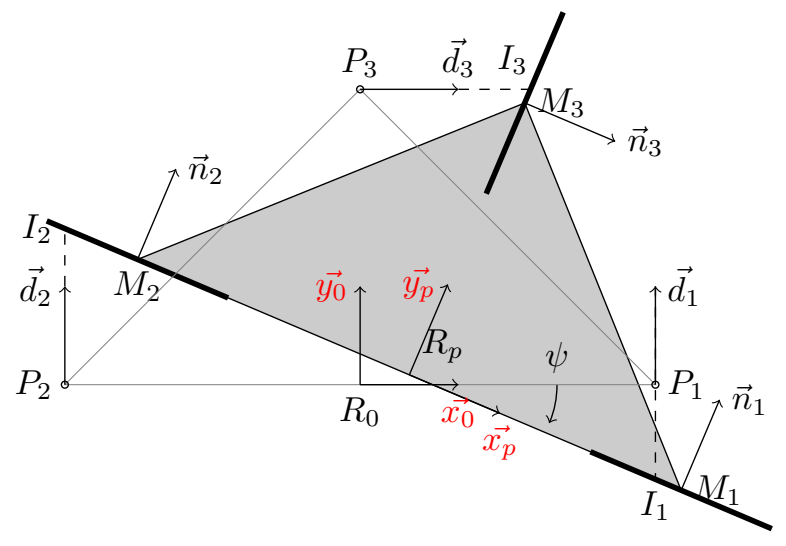

Fig. 8. Particular configuration of laser sensors (top view)

$$
\begin{aligned}
l & =\left(x_{n}-y_{n} \tan \psi\right) X+\left(x_{n} \tan \psi+y_{n}\right) Y \\
& +\left(y_{n} x_{P}-x_{n} y_{P}\right) \tan \psi
\end{aligned}
$$

The laser range sensors are placed on $P_{1}, P_{2}$ and $P_{3}$ with:

$$
P_{1}=\left[\begin{array}{l}
\delta \\
0
\end{array}\right]_{/ R_{0}}, P_{2}=\left[\begin{array}{l}
-\delta \\
0
\end{array}\right]_{/ R_{0}} \text { and } P_{3}=\left[\begin{array}{l}
0 \\
\delta
\end{array}\right]_{/ R_{0}}
$$

The system to be solved is:

$$
\left\{\begin{array}{l}
l_{1}=-X \tan \psi+Y+\delta \tan \psi \\
l_{2}=-\tan \psi X+Y-\delta \tan \psi \\
l_{3}=X+Y \tan \psi-\delta \tan \psi
\end{array}\right.
$$

and the solution is:

$$
\begin{gathered}
\tan \theta=\frac{l_{1}-l_{2}}{2 \delta} \\
X=\frac{\delta\left(2 \delta l_{1}-2 \delta l_{2}+4 \delta l_{3}-l_{1}{ }^{2}+l_{2}^{2}\right)}{4 \delta^{2}+l_{1}^{2}-2 l_{1} l_{2}+l_{2}{ }^{2}} \\
Y=\frac{\delta\left(l_{1}{ }^{2}-2 l_{1} l_{2}+2 l_{1} l_{3}+l_{2}{ }^{2}-2 l_{2} l_{3}+2 \delta l_{1}+2 \delta l_{2}\right)}{4 \delta^{2}+l_{1}{ }^{2}-2 l_{1} l_{2}+l_{2}{ }^{2}}
\end{gathered}
$$

\section{DYNAMIC MODEL OF THE PLATFORM}

The global aim or our study is to calculate a feedback control which allows force measurement without displacement of the platform (consign $=0$ ). The figure 9 describes the nature of the problem to be solved. The input vectors are the currents in the coils $\left(i_{1}, i_{2}, i_{3}\right)$ and the external efforts $\left(F^{e x t}, \xi_{\text {ext }}\right)$ applied to the platform (in the plane $x O y$ ) and considered as perturbations. The output vector is the platform position $(X, Y, \psi)$.

The state model is going to be established from the differential equations given by the platform dynamic behaviour in the plane $(x O y)$ :

$$
\vec{F}_{1}+\vec{F}_{2}+F_{/ R^{0}}^{\vec{v}}+F_{/ R^{0}}^{\overrightarrow{e x t}}=m \overrightarrow{\tilde{G}}_{/ R^{0}}
$$

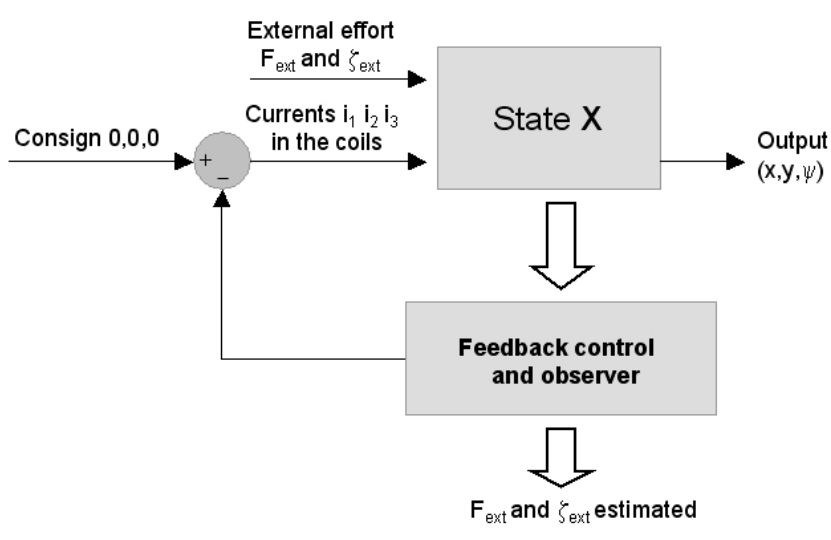

Fig. 9. Feedback control

in which $\overrightarrow{F^{e x t}}$ is the external force applied on the floating platform and $\overrightarrow{F^{v}}$ is the viscous friction force between the float and water. In case of small displacement we can write:

$$
\overrightarrow{F^{v}}=K_{v} \cdot \overrightarrow{\dot{G}}=\left[\begin{array}{cc}
K_{v}^{x} & 0 \\
0 & K_{v}^{y}
\end{array}\right] \cdot \overrightarrow{\dot{G}}
$$

The dynamic behaviour in case of circular movement is given by the following equation:

$$
\begin{aligned}
& \sum \mathcal{M} / G=J \dot{\Omega} \\
& \Rightarrow \vec{F}_{1} \wedge R_{p} \vec{M}_{1}+\vec{F}_{2} \wedge R_{p} \vec{M}_{2}+\zeta_{v}+\zeta_{\text {ext }}=J \dot{\Omega}
\end{aligned}
$$

with: $\Omega$ the instantaneous rotation vector.

\section{A. Evaluation of $\vec{F}_{1}$}

The force $\vec{F}_{1}$ applied on the moving magnet $M_{1}$ is given by the following expression:

$$
\overrightarrow{F_{1}}=F_{p_{1}}^{\overrightarrow{m a g}}+F_{b_{3}}^{\overrightarrow{e l e c}}
$$

where $F_{p_{1}}^{\overrightarrow{m a g}}$ is the magnetic force applied by $p_{1}$ on $M_{1}$ and $F_{b_{3}} \overrightarrow{e l e c}$ is the electromagnetic force applied by $b_{3}$ on $M_{1}$.

\section{B. Evaluation of $\vec{F}_{2}$}

The force $\vec{F}_{2}$ applied on the moving magnet $M_{2}$ is given by the following expression:

$$
\overrightarrow{F_{2}}=F_{p_{2}}^{\overrightarrow{m a g}}+F_{b_{1}}^{\overrightarrow{e l e c}}+F_{b_{2}}^{\overrightarrow{e l e c}}
$$

where $F_{p_{2}}^{\overrightarrow{m a g}}$ is the magnetic force applied by $p_{2}$ on $M_{2}$, $F_{b_{1}} \overrightarrow{e l e c}$ is the electromagnetic force applied by $b_{1}$ on $M_{2}$ and $F_{b_{2}}^{\text {elec }}$ is the electromagnetic force applied by $b_{2}$ on $M_{2}$.

The development of the equations (12) allows us to write:

$$
\begin{aligned}
m\left[\begin{array}{c}
\ddot{X} \\
\ddot{Y}
\end{array}\right]= & {\left[\begin{array}{cc}
-2 K_{m}^{x} & 0 \\
0 & -2 K_{m}^{y}
\end{array}\right]\left[\begin{array}{c}
X \\
Y
\end{array}\right]+\left[\begin{array}{cc}
-K_{v}^{x} & 0 \\
0 & -K_{v}^{y}
\end{array}\right]\left[\begin{array}{c}
\dot{X} \\
\dot{Y}
\end{array}\right] } \\
& +\left[\begin{array}{ccc}
-K_{e} & 0 & 0 \\
0 & K_{e} & K_{e}
\end{array}\right]\left[\begin{array}{c}
i_{1} \\
i_{2} \\
i_{3}
\end{array}\right]+\left[\begin{array}{l}
F_{x}^{e x t} \\
F_{y}^{e x t}
\end{array}\right]
\end{aligned}
$$

with $m$ the total platform mass. 
In case of circular movement projected on the $\vec{z}$ axis, we can write from the dynamic behaviour (14):

$$
\begin{aligned}
J_{\psi} \ddot{\psi}= & -K_{e} \cdot \delta\left(i_{1} \cdot \sin \psi+i_{2} \cdot \cos \psi-i_{3} \cdot \cos \psi\right)+2\left(\left(K_{m}^{x}\right.\right. \\
& \left.\left.-K_{m}^{y}\right) \cos \psi-K_{m}^{x}\right) \cdot \sin \psi \cdot \delta^{2}-K_{v}^{r o t} \dot{\psi}+\zeta_{\text {ext }}
\end{aligned}
$$

For very small $\psi$ we can write $\sin \psi=\psi, \cos \psi=1$ and $i_{1} \cdot \sin \psi \approx 0$, thus we have:

$$
J_{\psi} \ddot{\psi}=-K_{e} \cdot \delta\left(i_{2}-i_{3}\right)-2 K_{m}^{y} \psi \delta^{2}-K_{v}^{r o t} \dot{\psi}+\zeta_{\text {ext }}
$$

(17) and (18) give the linear state model suitable for small displacements:

$$
\begin{aligned}
{\left[\begin{array}{l}
\ddot{X} \\
\ddot{Y} \\
\ddot{\psi}
\end{array}\right]=} & {\left[\begin{array}{ccc}
-\frac{2 K_{m}^{x}}{m} & 0 & 0 \\
0 & -\frac{2 K_{m}^{y}}{m} & 0 \\
0 & 0 & -\frac{2 \delta^{2} \cdot K_{m}^{y}}{J}
\end{array}\right]\left[\begin{array}{l}
X \\
Y \\
\psi
\end{array}\right] } \\
+ & {\left[\begin{array}{ccc}
-\frac{K_{v}^{x}}{m} & 0 & 0 \\
0 & -\frac{K_{v}^{y}}{m} & 0 \\
0 & 0 & -\frac{K_{y}^{r o t}}{J_{\psi}}
\end{array}\right]\left[\begin{array}{c}
\dot{X} \\
\dot{Y} \\
\dot{\psi}
\end{array}\right] } \\
+ & {\left[\begin{array}{ccc}
-\frac{K_{e}}{m} & 0 & 0 \\
0 & \frac{K_{e}}{m} & \frac{K_{e}}{m} \\
0 & -\frac{\delta \cdot K_{e}}{J_{\psi}} & \frac{\delta \cdot K_{e}}{J_{\psi}}
\end{array}\right]\left[\begin{array}{c}
i_{1} \\
i_{2} \\
i_{3}
\end{array}\right] } \\
+ & {\left[\begin{array}{ccc}
\frac{1}{m} & 0 & 0 \\
0 & \frac{1}{m} & 0 \\
0 & 0 & \frac{1}{J \psi}
\end{array}\right]\left[\begin{array}{c}
F_{x}^{\text {ext }} \\
F_{y}^{\text {ext }} \\
\zeta_{\text {ext }}
\end{array}\right] }
\end{aligned}
$$

and the state variable representation is:

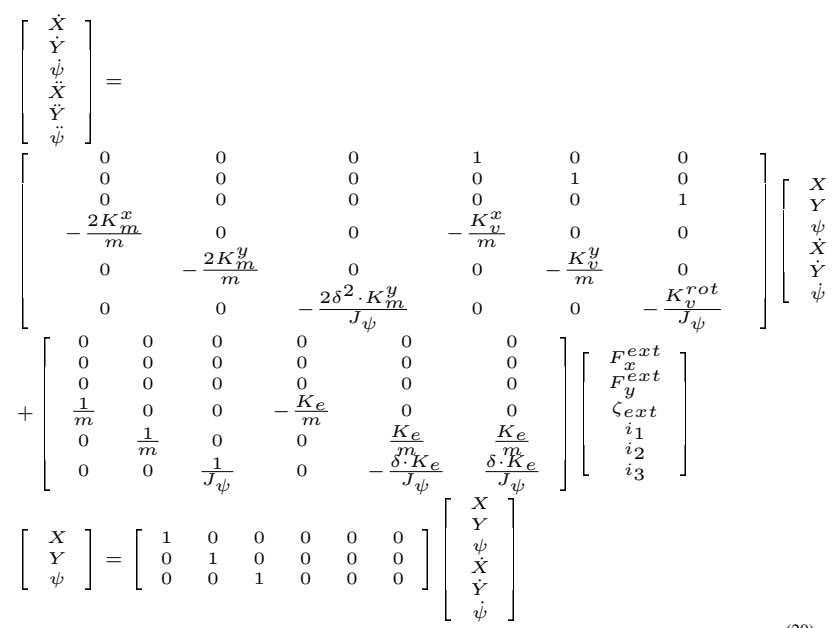

\section{MODEL VALIDATION}

Thanks to the dynamic equations, we have build a $2 \mathrm{D}$ analytical model programmed in $\mathrm{C}++$ and embedded in Matlab/Simulink. This model allows us to simulate the open loop response of the platform to an external applied force. The figure 10 shows the step responses, for a positive current $i_{1}=0.4 \mathrm{~A}$, of the linear 2D analytical model and the complete $3 \mathrm{D}$ analytical model. Instead of using linear equations (1) and (2), the 3D non linear model is based on the calculus of the magnetic inductions generated by the $p_{i}$ magnets and the $b_{i}$ coils and applied on the $M_{i}$ magnets. This allows the determination of the magnetic forces $F_{p_{i}}^{\overrightarrow{m a g}}$ and the electromagnetic forces $F_{b_{i}}^{\overrightarrow{e l e c}}$ applied to the platform. The complete 3D behavior of the platform is performed by integrating equations (12) and (14) with simulink.
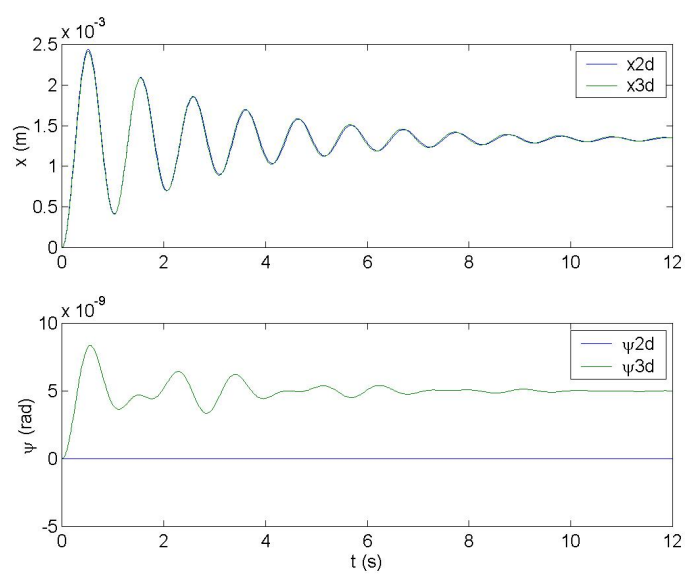

Fig. 10. Responses along $\vec{x}$ and $\vec{\psi}$ of the two 2D and 3D analytical models for a step current $i_{1}=0.4$

By comparison of the two models resulting curves, the linear 2D analytical approach only makes small changes in the temporal behaviour of the system. Therefore, the linear state model (19) is sufficient to calculate a controler if currents in coils are below $0.4 \mathrm{~A}$.

\section{CONCLUSION}

In this paper we have presented a $2 \mathrm{D}$ modelling of a six degrees of freedom platform used as the sensing part of a micro and nanoforce sensor based on a floating-magnetic principle. The particular configuration used in order to simplify the differential equations which govern the movement of the platform allowed us to build a 2D linear model of this micro and nanoforce sensor. This linear model and the state variable representation presented in this paper will be essential for the control of our multivariable system in order to achieve force measurement without deplacement of the sensing part. In this paper we have also presented the way to calculate the platform position with the use of three laser range sensors mounted in a particular configuration.

\section{REFERENCES}

[1] G. Asch, "Les capteurs en instrumentation industrielle," Dunod, 1999.

[2] M. Boukallel, J. Abadie, and E. Piat, "Levitated micro-nano force sensor using diamagnetic levitation," Proc. of the IEEE International Conference of Robotics and Automation, pp. 3219-3224, september 2003.

[3] M. Boukallel, E. Piat, and J. Abadie, "Passive diamagnetic levitation: theoretical foundations and application to the design of a micro-nano force sensor," Proceedings of the 2003 IEEE/RSJ intl. Conference on Intelligent Robots and Systems Las Vegas, Nevada, pp. 1062-1067, October 2003.

[4] A. Cherry, J. Abadie, and E. Piat, "Microforce sensor for microbiological applications based on a floating-magnetic principle," IEEE International Conference on robotic and automation, April 2007.

[5] S. Earnshaw, "On the nature of the molecular forces," trans. Cambridge phil.Soc., pp. 97-112, 1842.

[6] Z. Lu, P. Chen, and W. Lin, "Force sensing and control in micromanipulation," TO appear in IEEE SMC part C.

[7] Y. Shengyuan and S. Taher, "Micromachined force sensors for study of cell mechanics," Review of scientific instruments 76, 044301, 2005.

[8] Y. Sun, W. Kai-Tak, K. Roberts, J. Bischof, and J. Bradley, "Mechanical property characterization of mouse zona pellucida," IEEE transactions on nanobioscience, vol. 2, no. 4, pp. 279-285, December 2003. 